\title{
Collective stringlike motion of semiflexible filamentous particles in columnar liquid crystalline phases
}

\author{
Saber Naderi ${ }^{1,2, *}$ and Paul van der Schoot ${ }^{1,3}$ \\ ${ }^{1}$ Faculteit Technische Natuurkunde, Technische Universiteit Eindhoven, Postbus 513, 5600 MB Eindhoven, The Netherlands \\ ${ }^{2}$ Dutch Polymer Institute, P.O. Box 902, 5600 AX Eindhoven, The Netherlands \\ ${ }^{3}$ Instituut voor Theoretische Fysica, Universiteit Utrecht, Leuvenlaan 4, 3584 CE Utrecht, The Netherlands
}

(Received 9 July 2013; published 24 September 2013)

\begin{abstract}
We study, by means of Brownian dynamics simulations, heterogeneous dynamics in a dense columnar phase of monodisperse hard filamentous particles, and find that in a background of barely moving particles, some particles occasionally engage in a fast coherent string-type motion similar to what is observed in glassy states of isometric particles. This fast motion is triggered by the exchange of particles between two or more columns at different positions in the columns, which leads to sudden displacement of particles between these positions. The distribution of particle displacements shows a pronounced peak at one particle length. We perform our simulations with particles of different persistence lengths and find that for more flexible particles, the number of jump events increases. As the number of particles in the columns increases with system size for a given linear fraction of particles in the columns, the peak in the distribution becomes wider and, for sufficiently large systems, the peak disappears completely. This is associated with the increase in the magnitude of fluctuations in the motion of particles as the system size increases. Our simulation results explain recent experimental observations on single-particle motion in dense columnar phases in aqueous dispersions of filamentous virus particles.
\end{abstract}

DOI: 10.1103/PhysRevE.88.032307

PACS number(s): 82.70.Dd, 61.30.Cz, 82.20.Wt

\section{INTRODUCTION}

Liquid crystalline phases formed in suspensions of highly anisometric, i.e., elongated or flat, colloidal particles have been the subject of intensive study over the past few decades [1-6]. Among these particles, filamentous virus particles, such as tobacco mosaic virus (TMV) and $f d$ virus, are particularly interesting because, unlike most other types of colloidal particle, they are very monodisperse [4], which makes them a suitable model system to compare with theory and simulation. (See, however, the recent work of the group of Van Blaaderen on monodisperse silica rods [7].) Onsager [8] showed that the isotropic-to-nematic phase transition in dispersions of long hard rods, i.e., rods interacting via a harshly repulsive, steric interaction, can be driven by entropy alone. Indeed, aqueous dispersions of $f d$ virus, TMV, and DNA do form a (chiral) nematic phase at sufficiently large concentration [9-12]. It has also been predicted (based on an Onsager-like theory) that at large packing fraction, parallel hard rods form a smectic-A phase [13]. Frenkel and collaborators did indeed find a smectic phase of hard rods in their simulations, showing that excluded-volume effects can give rise to complex phases [14]. A stable smectic-A phase in dispersions of $f d$ virus, TMV, highly monodisperse poly( $\gamma$-benzyl L-glutamate), and silica rods has been observed experimentally [7,15-17].

Recently, Grelet found that filamentous $f d$ virus particles of length and width 880 and $7 \mathrm{~nm}$, respectively, and a persistence length of about $2.8 \mu \mathrm{m}$, self-organize into a columnar phase in water at high concentrations [18]. This columnar phase has a polydomain ("powder") structure characterized by grain boundaries. The size of the domains, which can be estimated from optical textures obtained by polarizing microscopy, measures from about 10 to 100 virus particle lengths. X-ray

"m.s.naderi@tue.nl scattering patterns of monodomains turn out to show a sixfold symmetry with peaks that have substantial tails, indicative of a hexatic rather than hexagonal order. The hexatic structure is characterized by quasi-long-range sixfold orientational and a short-range translational order resulting from the presence of topological defects, presumably caused by the chiral nature of the particles $[19,20]$.

The reason why $f d$ virus particles form a columnar phase at high concentrations remains poorly understood. Theoretically, particle bending flexibility can destabilize the smectic phase and it has been suggested that for sufficiently flexible particles, this may lead to the formation of a columnar phase $[21,22]$. However, in computer simulations of wormlike particles with varying flexibility, no indication of a stable columnar phase was found, at least for hard particles with aspect ratios up to 5.8 [23]. By applying a bifurcation analysis to the Onsager free energy for charged rods in strongly nematic states, Wensink found that a hexagonal columnar phase suppresses the smecticA phase if colloidal rods are perfectly parallel and charged, and the Debye screening length is sufficiently large [24]. He also observed this in simulations using a Yukawa site model, where a strong alignment potential was applied to the particles. Interestingly, the hexagonal symmetry of the columnar phase vanished in the absence of this alignment field. Another study showed that for monodisperse parallel hard spherocylinders, the formation of the columnar phase strongly depends upon the system size [25]. A meta-stable columnar phase (with respect to a crystal phase) was found in simulations of infinitely long spherocylinders [26]. In computer simulations, a columnar phase is usually stabilized by making use of a length polydispersity of the particles $[27,28]$. It has also been observed experimentally that polydisperse particles form a columnar phase at sufficiently high concentrations [29].

Recent advances in experimental techniques have made it possible to probe the Brownian motion of individual particles in real space in dense liquid crystalline phases, including 
the nematic, smectic, and columnar phases. Käs and coworkers studied bending fluctuations and self-diffusion of actin filaments in dilute, semidilute, and concentrated F-actin solutions [30]. In 2003, the arrangement and dynamics of monodisperse $\beta$-FeOOH, selenium (Se), and nickel dimethylglyoxim (NDG) rods were studied in real space by optical microscopy [31]. Later, self-diffusion of filamentous virus particles in the nematic phase was studied by means of fluorescent microscopy techniques [32]. It was found, for instance, that the self-diffusivity along the main axis of the particles speeds up in the nematic phase compared to that in the isotropic phase. In the smectic phase, diffusion of particles along the main axis has been found to be a kind of hopping-type diffusion between smectic layers [33-35], dictated by the temporary caging of particles by their direct neighbors and by the permanent self-consistent molecular field that they experience due to the presence of all the other particles [36]. Smectic phases of monodisperse silica rods also showed a hopping-type interlayer diffusive motion, albeit that in this case the layer-to-layer diffusion is slower than the in-layer diffusion [7,37]. This unusual type of diffusion of particles in the smectic phase has been observed in computer simulations as well, highlighting the highly correlated nature of particle motion in congested phases [38-40].

Due to the layered structure of the smectic phase, it is not surprising that a hopping-type diffusion between the layers has been observed. Interestingly, discrete jumps have also been found by Grelet et al. in the columnar phase of $f d$ virus particles [41]. In this study, a small fraction of the virus particles were fluorescently labeled so that the position of the particles could be recorded over time. Their displacement along the director of the columnar phase, i.e., parallel to the column axes, shows sudden jumplike displacement similar to that found in the smectic phase. The distribution of jump lengths obtained from these experiments is bimodal with peaks on a half and a full particle length. This shows that in contrast to the jump length distribution in the smectic phase, which is peaked at about a full particle length, in the columnar phase there are two types of jump with characteristic lengths of a half or a full particle length.

One plausible explanation is that the full jumps may be associated with particle overtaking events and the half jumps are due to the reequilibration of particles in a column following a jump of a particle out of the column, e.g., into a defect. To investigate this, we recently performed Brownian dynamics simulations of hard particles confined by a cylindrically symmetric Gaussian potential that mimics the self-consistent field that particles in a column experience due to the presence of the other particles [42]. Our simulation results showed that overtaking events present themselves as full jumps only if the size of a column is sufficiently small. This is because in large systems the jumps are washed out by the large fluctuations in the motion of particles. Hence, the reason why these jumps are observed experimentally must be connected with the polydomain structure of the columnar phase of $f d$ virus particles. We also performed simulations where a particle from the middle of a column is removed and the positions of its neighbors are tracked, and found that during reequilibration of the system, these particles move very fast compared to the self-diffusion time of single particles. Displacement of the neighbors after reequilibration turned out to be (on average) about a half particle length, confirming our hypothesis.

In our previous simulations, we considered only a single column so the influence of inter-column interactions was not taken into account. Here, we perform Brownian dynamics simulations of a hexagonal columnar phase of hard semiflexible filamentous particles to investigate other possible mechanisms that lead to half and full jumps. In order to prevent formation of the smectic phase, which appears to be the most stable phase for our particles at concentrations below which they crystallize and concentrations above which they form a nematic phase [23], we apply a superposition of three periodic potentials in two dimensions. We find an interesting correlated closed-loop-like (or "vortexlike") motion of particles, similar to those observed in glassy states of spherical particles [43]. Among those particles involved in the correlated motion, some change columns at different positions and others stay in the same column engaging in full-length jumps. We perform simulations with two types of particles of equal length but different persistence length, and find that the number of jump events increases with increasing the particles' internal flexibility. This is in line with experimental observations on the wild-type $f d$ virus and the mutant $f d Y 21 M$ virus, the latter of which is much stiffer [41].

The remainder of this paper is organized as follows. In Sec. II, we describe our simulation model and the way we analyze our simulation data. Section III is devoted to describing our finding of correlated stringlike displacement events involving particles in up to six columns. The influence of system size on these events is discussed in Sec. IV, where we also present our results for the Van Hove self-correlation function. In Sec. V, we discuss the effect of particle bending flexibility on the motion of particles. We end our paper with conclusions and outlook in Sec. VI.

\section{MODEL AND SIMULATIONS}

In our Brownian dynamics simulations, there are $N$ chainlike particles, each containing a linear array of $n=9$ beads. Within each particle, adjacent beads are connected to each other via a harmonic bond potential of the form $U_{\text {bond }}(r)=k_{b}\left(r-l_{b}\right)^{2}$, and each three neighboring beads are in addition linked by a harmonic bending potential $U_{\text {bend }}(\theta)=$ $k_{a}(\theta-\pi)^{2}$, where $r$ is the center-to-center distance between pairs of beads and $l_{b}=0.6 \sigma$ is the equilibrium bond length with $\sigma$ the size (diameter) of the beads and $\theta$ the angle formed by the two bonds that link the three beads to each other. Furthermore, $k_{b}$ and $k_{a}$ are the strengths of the bond and bend potentials, respectively. The total length $L$ of our particles is $L=(n-1) l_{b}+\sigma$. To make certain that bond lengths are essentially fixed during the simulations, we choose a large value for the strength of the bond potential, $k_{b}=50 k_{B} T / \sigma^{2}$, where $k_{B} T$ is the thermal energy with $k_{B}$ the Boltzmann constant and $T$ the absolute temperature. The persistence length of our particles $L_{p}$ is related to $k_{a}$ via $L_{p}=2 k_{a} l_{b} / k_{B} T[44,45]$, at least in the limit where $n \rightarrow \infty$ and $k_{b} l_{b}^{2} / k_{B} T \gg 1$. In our simulations, we focus attention on ratios $L_{c} / L_{p}=0.09-0.31$ of the contour length $L_{c}=(n-1) l_{b}$ of our particles in the stiff bond limit that we are considering and their persistence length $L_{p}$. This allows us to investigate the effect of particle 
flexibility on the kinetics. As we shall see, particle flexibility has a large impact on their long-time diffusive motion.

All beads, excluding those that are direct neighbors in a chain, interact with each other through the repulsive part of a shifted Lennard-Jones potential,

$$
U_{i j}(r)= \begin{cases}4 \epsilon\left[\left(\frac{\sigma}{r}\right)^{12}-\left(\frac{\sigma}{r}\right)^{6}+\frac{1}{4}\right] & \text { if } \quad r \leqslant 2^{\frac{1}{6}} \sigma \\ 0 & \text { if } \quad r>2^{\frac{1}{6}} \sigma,\end{cases}
$$

where $\epsilon$ is the strength of the interaction potential, which in our simulations is equal to the thermal energy, so $\epsilon=k_{B} T$, and $r$ is again the distance between the centers of mass of the beads. This potential mimics the soft screened electrostatic repulsion that acts between the charge-stabilized $f d$ virus particles in the experiments of Grelet and collaborators [18,41].

In order to prevent the formation of a smectic phase in our simulations and to mimic the self-consistent field that particles experience in the hexagonal columnar phase, we apply an external potential to all the particles (beads). This potential is a superposition of three periodic potentials in two dimensions,

$$
\begin{aligned}
U_{\text {ext }}(x, y)= & \frac{-k}{2}\left\{\cos (q x)+\cos \left[\frac{q}{2}(\sqrt{3} y-x)\right]\right. \\
& \left.+\cos \left[\frac{q}{2}(\sqrt{3} y+x)\right]\right\},
\end{aligned}
$$

where $k$ is the strength of the external potential and $q$ is defined as $q=2 \pi / d$ where $d$ is the equilibrium column-to-column distance. Note that this column-to-column distance also affects the bending fluctuations of our particles. This is because each particle is surrounded by six other particles and it is effectively confined in a tube with a diameter of $d$. It introduces a different length scale, the so-called deflection length $\lambda$, and is related to the persistent length of our particles, $L_{p}$, and the columnto-column distance $d$ by $\lambda=L_{p}^{1 / 3} d^{2 / 3}$ [46,47]. Our model for semiflexible chains is sensible only in the regime where the deflection length is larger than the bond length $l_{b}$. In our simulations, this is always true.

To investigate the effect of polydomain structure of the columnar phase and the domain size on the motion of particles, we perform simulations on three system sizes with $N=12$, 50, and 200 particles in each column. There are 100 columns in the simulation box, so our system size varies from 1200 to 20000 rods. Initially, in each column, particles are positioned equidistantly on a line with a linear fraction $\psi$. The position of this line in the plane perpendicular to the columns is determined by the position of the minima of the external field. We apply reflecting boundary conditions along the main axes of the columns (parallel to the director) and, in the direction perpendicular to the director, we apply periodic boundary conditions. To suppress any smectic layering of particles along the director, which may result from flat boundaries, we place two fixed particles at the two ends of each column. These two particles contain $n_{1}$ and $n_{2}$ beads with the condition that $n_{1}+n_{2}=n$ where $n=9$ is the number of beads of the mobile particles in our system and $n_{1}$ is chosen randomly for each column (see Fig. 1).

We cannot exclude the possibility that the hexagonally ordered pinned particles by themselves stabilize a columnar phase even without the external field, at least if the system

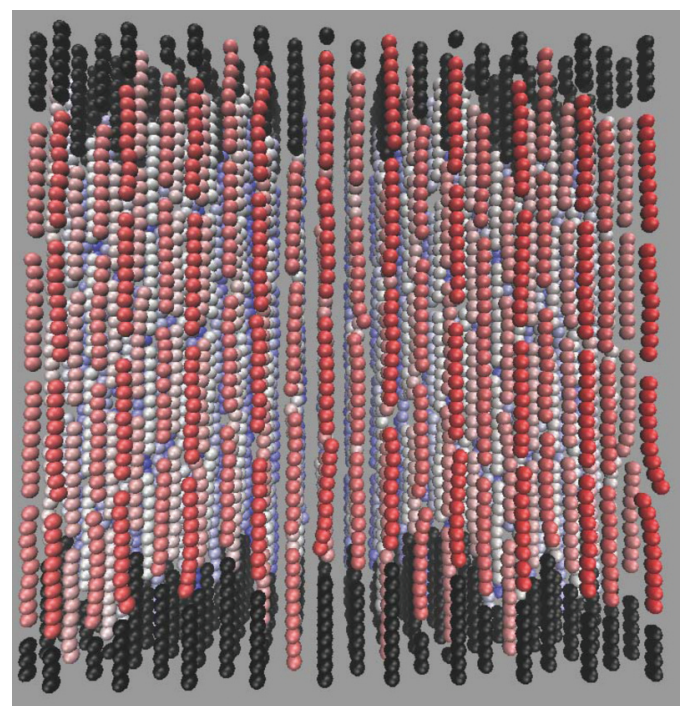

FIG. 1. (Color online) (a) Snapshot of a simulation box with 100 columns containing five particles each. Each particle is made up of nine beads. Black particles at the top and the bottom of the simulation box are fixed. These particles prevent smectic layering of other particles, which are here colored according to their position in the simulation box from red to blue. The equilibrium column-to-column distance $d$ is equal to $1.75 \sigma$, where $\sigma$ is the diameter of the beads; the strength of the external potential enforcing a hexagonal arrangement of the particles in the columns is $k=1.5 k_{B} T$. See also the main text.

size is small enough. This presumably requires careful tuning of the intercolumn distance, which we intend to address in follow-up work. To keep full control over intercolumnar distances, we prefer to impose the external field in this work.

Our Brownian dynamics simulations are performed with the LAMMPS molecular dynamics package [48]. We add a custom potential to the LAMMPS code and implement the external potential of Eq. (2) in the code. We choose a time step of $5 \times 10^{-3} t^{*}$ where $t^{*}$ is the unit of time, set by the self-diffusion constant of a single bead $D_{b}=\sigma^{2} / t^{*}$. A particle made up of $n$ beads diffuses with a self-diffusion constant of $D=D_{b} / n$, at least in the free-draining limit in which we are operating [49]. The self-diffusion time of a particle is defined as $\tau_{D}=L^{2} / D$ where $L=(n-1) l_{b}+\sigma$ is the length and $D=D_{b} / n$ is the self-diffusivity of a particle. The self-diffusion time increases with the third power of the number of beads in each particle, $n$, i.e., the computational cost of a simulation for a given simulation time (in terms of $\tau_{D}$ ) increases with the third power of $n$, apart from the fact that for a given number of rods, the total number of beads also increases with increasing $n$. Hence, to keep our simulations computationally feasible, particles in the simulations are made up of nine beads with aspect ratio of 5.8, which is much smaller than that of the $f d$ virus that measures about 100 .

To analyze our simulation data, we calculate the distribution of jump lengths and the self-part of the Van Hove function for each simulation. The self-part of the Van Hove function, $G_{s}(\mathbf{r}, t)$ [50], is a measure of the probability of finding a particle at a distance $\mathbf{r}$ from its position at some initial time, after a 
time interval of $t$. It is defined as [50]

$$
G_{s}(\mathbf{r}, t)=\frac{1}{N}\left\langle\sum_{i=1}^{N} \delta\left[\mathbf{r}+\mathbf{r}_{i}\left(t_{0}\right)-\mathbf{r}_{i}\left(t+t_{0}\right)\right]\right\rangle,
$$

where $N$ is the number of particles and the angular brackets imply an ensemble average. In order to separately study the diffusion of the particles along and perpendicular to the director, we decompose the self-part of the Van Hove function into two components: a component along the director, i.e., the $z$ axis,

$$
G_{s}^{\|}(z, t)=\frac{1}{N}\left\langle\sum_{i=1}^{N} \delta\left[z+z_{i}\left(t_{0}\right)-z_{i}\left(t+t_{0}\right)\right]\right\rangle,
$$

and a component perpendicular to it,

$$
G_{s}^{\perp}(R, t)=\frac{1}{N}\left\langle\sum_{i=1}^{N} \delta\left[R+R_{i}\left(t_{0}\right)-R_{i}\left(t+t_{0}\right)\right]\right\rangle,
$$

where $R$ denotes the magnitude of the projection of $\mathbf{r}$ in the $x y$ plane.

To obtain the distribution of jump lengths, we developed an algorithm to detect when and where jumps occur in our simulations. We divide the entire simulation time frame into intervals of $500 t^{*}$ and calculate the average position of each particle and its standard deviation in each time interval. We also calculate the mean standard deviation for each particle in each simulation by averaging the standard deviations over all time intervals. If the ratio of the standard deviation of the position of a particle along the director at a time interval to the average standard deviation of that particle exceeds a value of 3.0, we subtract the average position of this particle before and after this time interval, and record it as a jump length. This way, we obtain a set of jump lengths from which we calculate the jump length distributions.

\section{CORRELATED MOTION IN THE COLUMNAR PHASE}

As mentioned earlier, we apply an external potential of the form of Eq. (2) to all beads in order to stabilize the columnar phase. The free energy barrier that a particle needs to overcome to jump to another column is determined by the strength of this external potential $k$, the column-to-column distance $d$, and particle-particle interactions, i.e., the density. We choose a value for $d$ that is comparable to the experimental values for the columnar phase formed by $f d$ virus particles. For the $f d$ virus system, the column-to-column distance measures about two to three times the diameter of the particles [41]. However, since the aspect ratio of our particles is much smaller than that of the $f d$ virus, our particles have more rotational freedom for the same value of column-to-column distance. Therefore, we choose the column-to-column distance, $d=1.75 \sigma$, where $\sigma$ is the width of our particles, which is slightly smaller than the experimental value but at the same time sufficiently large to allow particles to overtake each other within a column.

In order to obtain a reasonable value for the strength of the external potential $k$, we tune this parameter to obtain values for the free energy barrier between columns that are comparable to those obtained from simulations done by Belli and co-workers [28]. To obtain the free energy barrier between
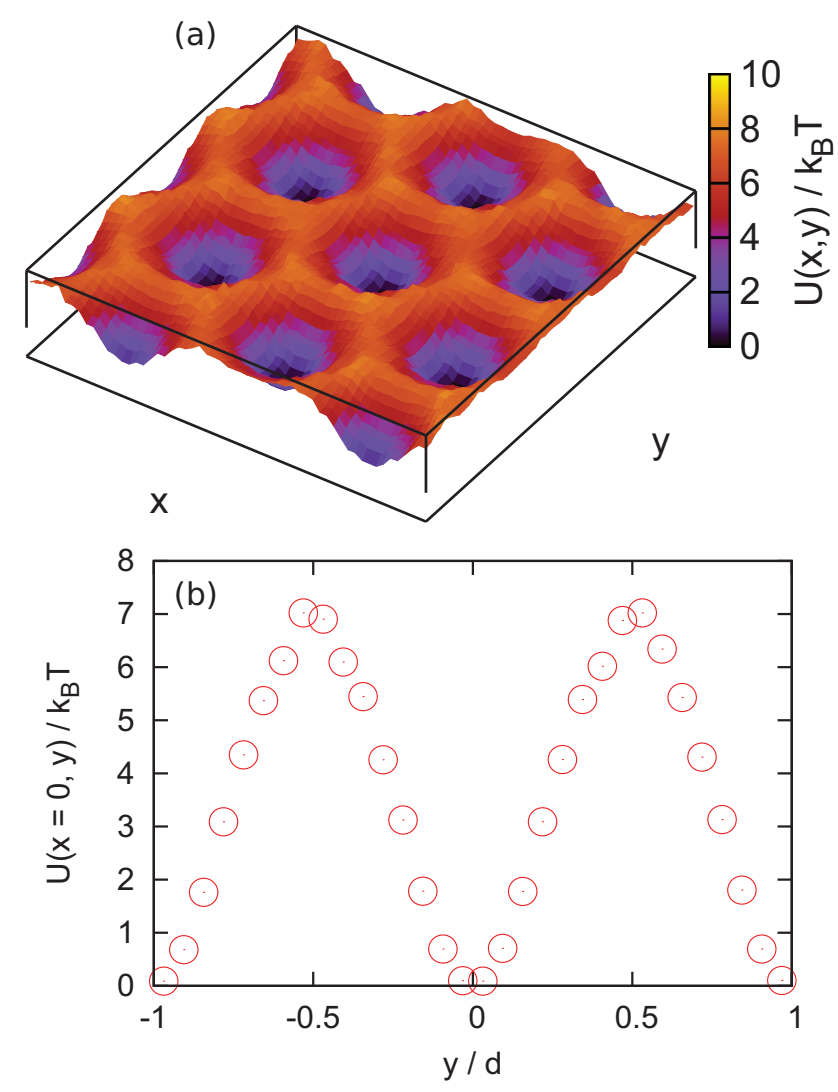

FIG. 2. (Color online) (a) Mean-field potential, $U(x, y)$, that the semiflexible filamentous particles in our simulations experience in the direction perpendicular to the director, in units of thermal energy $k_{B} T$. Length to persistence length ratio of each particle that contains nine beads equals $L / L_{p}=0.31$. A superposition of three external periodic potentials is applied to all beads. Here, the strength of the external potential is $k=1.5 k_{B} T$ and the average column-to-column distance $d$ is equal to $1.75 \sigma$, where $\sigma$ is the diameter of a bead. The average number of particles in each column is 12 with an average linear fraction of $\psi=0.95$. There are 100 columns in the simulation box. (b) Cross section of the mean-field potential at $x=0$.

columns, we calculate the mean-field potential that particles experience in the direction perpendicular to the director. This potential is defined as $U(x, y)=-k_{B} T \ln p(x, y)$ apart form an uninteresting constant, where $p(x, y)$ is the probability of finding a particle at position $(x, y)$. After examining different values for $k$, we find that the height of the free energy barrier for $k=1.5 k_{B} T, d=1.75 \sigma$, and initial linear fraction of $\psi=0.95$ in each column is about $7 k_{B} T$ (Fig. 2), which is about the same as the free energy barriers obtained from simulations by Belli and co-workers [28].

We perform our Brownian dynamics simulations with these parameters in order to see whether particles are able to overtake each other and engage in full jumps. In our simulations, there are 100 columns in the simulation box and, for the simulations we focus on in this section, each column contains, on average, 12 particles. Initially, these 12 particles are placed equidistantly with a linear fraction of $\psi=0.95$ on the central line of each column, which corresponds to one of the minima of the two-dimensional external field. We find that overtaking events are extremely rare in our simulations, which is not 


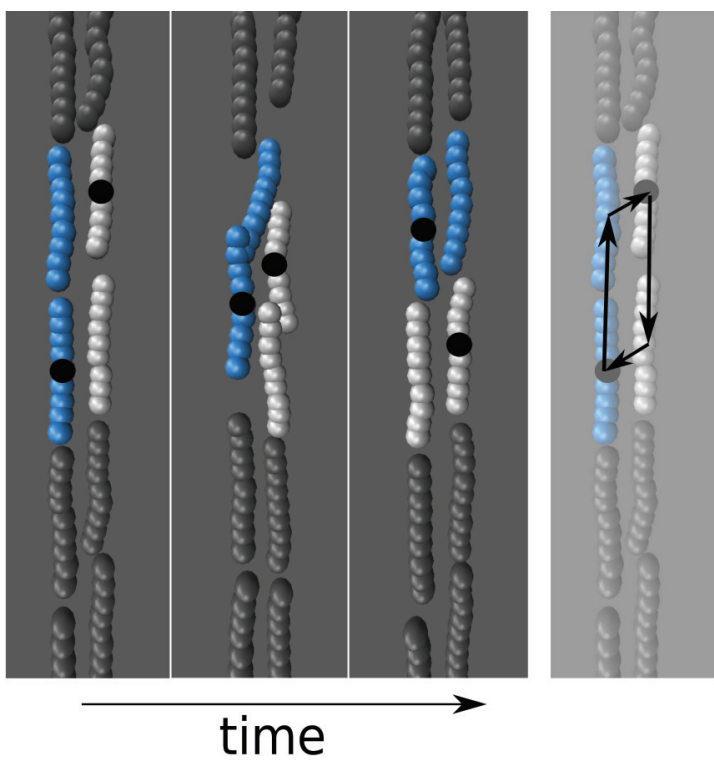

FIG. 3. (Color online) Three snapshots of parts of two columns in which some particles, colored in blue (light gray) and white, engage in a loop-type motion (the three panels on the left side). Two of these particles (marked by black beads) make full jumps while staying in a column. The other two particles exchange columns. Gray particles are not involved in this motion. In the panel on the right side, displacement of each particle during the loop-type motion is indicated by an arrow. Here, there are 100 columns in the simulation box. Each column contains 12 particles and each particle consists of nine beads with a length to persistence length ratio of $L / L_{p}=0.31$. The strength of the external potential is $k=1.5 k_{B} T$ and the equilibrium column-tocolumn distance $d$ is equal to $1.75 \sigma$.

surprising because the column-to-column distance is small and intercolumn interactions restrict the motion of particles in the direction perpendicular to the axes.

Instead, we find another mechanism that leads to fulllength jumps and that involves correlated loop-type motion of particles reminiscent of a vortex. We observe that in a background of barely moving particles, some particles in two, three, four, and so on columns simultaneously exchange columns at different positions and all particles in between them undergo a fast displacement that measures about a full particle length. The vortices are extremely rare and we have never observed more than one at the same time.

Shown in Fig. 3 are three snapshots of two columns in which this remarkable kind of correlated motion occurs. Note that in this particular example, only two columns are involved, but normally in our simulations more than two columns are involved. In the figure, four particles engage in the loop-type motion, two of which exchange columns at different positions and trigger the correlated motion. The other two stay in their columns and suddenly make full-length jumps, a number less than what we find on average in our simulations. On average, about four to five particles per column are involved. The heterogeneous dynamics involving fast correlated motion of a small fraction of otherwise almost static particles is not dissimilar to that found in colloidal glasses of spherical particles [43].

\section{DOMAIN SIZE EFFECTS}

As stated in Sec. I, optical textures obtained from experiments on the columnar phase formed by $f d$ virus particles in water shows that this phase has a polydomain structure with grain boundaries [18]. From this optical texture, the size of these domains can be estimated to be of the order of 10 particle lengths. This rather small domain size may affect the observed motion of particles in the columnar phase. To investigate this, we perform simulations with $N=12,50$, and 200 particles in each column. There are 100 columns in these simulations with an equilibrium column-to-column distance of $d=1.75 \sigma$ and an overall packing fraction of 28\%. Shown in Figs. 4(a)-4(c) is the displacement of three particles along the director corresponding to these three system sizes. As can be seen, the magnitude of the fluctuations in the trace of the particles increases with increasing the number of particles per column. For the case of $N=200$ particles per column, the magnitude of the fluctuations is of the order of the size of a particle and full jumps are washed out by the fluctuations, i.e., are no longer discernible. This can be also seen in Figs. 4(d)-4(f) where the distribution of jump lengths is shown. For the simulation with $N=12$ particles in each column, there is a sharp peak on $\Delta z / L=1.0$. The distribution becomes wider around $\Delta z / L=1.0$ as the number of particles

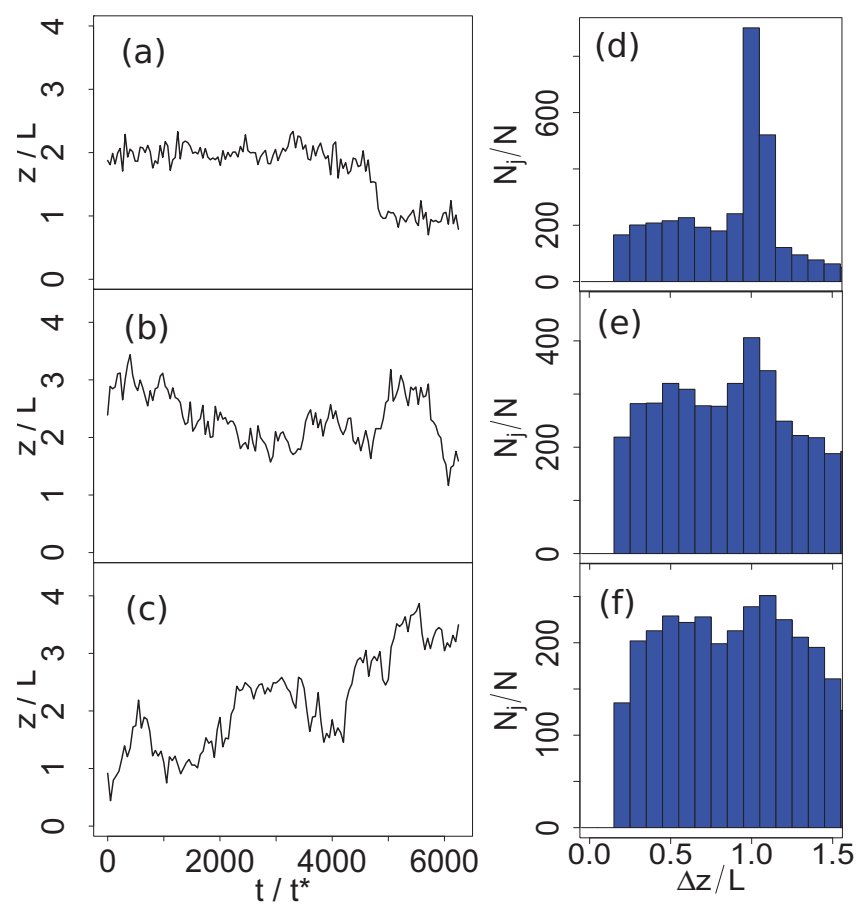

FIG. 4. (Color online) Displacement of particles along the director $z$ (left) and distribution of jump lengths (right) obtained from simulations with (a), (d) $N=12$, (b), (e) $N=50$, and (c), (f) $N=200$ particles per column. $N_{j}$ is the number of particles that jump with a certain jump length $\Delta z / L$. Here, $L$ is the length of a particle and $t^{*}$ is the simulation time unit, defined as $t^{*}=\sigma^{2} / D_{b}$ where $\sigma$ and $D_{b}$ are the diameter and self-diffusion constant of a single bead. There are 100 columns in the simulation boxes with column-to-column distance of $d=1.75 \sigma$. The strength of the external potential is $k=1.5 k_{B} T$. Each particle contains nine beads and has a length to persistence length ratio of $L / L_{p}=0.31$. 

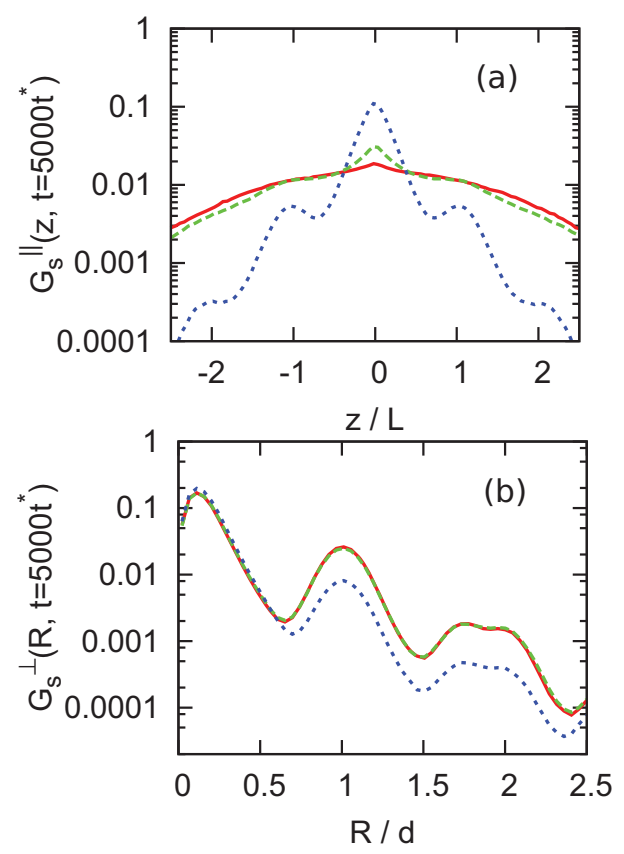

FIG. 5. (Color online) Components of the self-part of the Van Hove function that are (a) parallel, $G_{s}^{\|}(z, t)$, and (b) perpendicular, $G_{s}^{\perp}(R, t)$, to the column axes after a time interval of $t=5000 t^{*}$ obtained from simulations with $N=200$ (red solid line), $N=50$ (green dashed line), and $N=12$ (blue dotted line) particles per column. The average linear fraction of particles in each column is $\psi=0.95$. Here, $L$ is the length of a particle and $t^{*}$ is the simulation time unit. There are 100 columns in the simulation boxes with column-to-column distance of $d=1.75 \sigma$. The strength of the external potential is $k=1.5 k_{B} T$. Each particle contains nine beads and has a length to persistence length ratio of $L / L_{p}=0.31$.

per column increases, and for the simulation with $N=200$, the peak has nearly disappeared due to the strong fluctuations in the columns. There is an indication of a broadly distributed fraction of particles engaging in half jumps.

More information on the motion of particles along (and perpendicular to) the column axes can be obtained from the self-part of the Van Hove function. Shown in Fig. 5(a) is the self-part of the Van Hove function for the displacement of particles along the director $z$, after a time interval of $t=5000 t^{*}$ for the above-mentioned systems with $N=12,50$, and 200 particles per column. For the smallest system, peaks appear on the integer values of $z / L$ where $L$ is one particle length. This is similar to what has been observed in smectic-A phases formed by filamentous particles [7,33,37]. Due to the layered structure of the smectic-A phase, particles experience a periodic self-consistent molecular field perpendicular to the layers and therefore they mostly rattle around their position in a layer and occasionally jump from one layer to another. Because the layer-to-layer distance in this phase is about one particle length [33], this rattling and jumping motion presents itself as peaks in the self-part of the Van Hove function similar to those we observe in our simulations. The mechanism of the rattling and jumping motion that also presents itself in our simulations on the hexagonal phase is different from that in the smectic-A phase. As mentioned earlier, in our simulations, particles in two or more neighboring columns engage in a loop-type correlated motion. During this correlated motion, some particles stay in their columns and make jumps with a jump length of about one particle length. The jump length is dictated by the particle-to-particle distance along the columns, which due to the high linear fraction of particles in the columns is about one particle length.

As mentioned earlier, for large systems, the full-length jumps are washed out by fluctuations in the motion of particles. This expresses itself also in the self-part of the Van Hove correlator presented in Fig. 5, where the sharpness of the peaks on integer values of $z / L$ decreases as the number of particles per column increases. The intracolumn fluctuations, on the other hand, also enhance the column-to-column jumps because as the size of fluctuations increases, the probability of the formation of a sufficiently large void in a column to receive a jumper from a neighboring column increases. This can be seen in Fig. 5(b), where the self-part of the Van Hove function in the direction perpendicular to the columns axes is shown. For all values of the number of particles per column, $N$, there is a peak on $R / d=1$, which indicates that the rattling and jumping motion also occurs in this direction, from one column to another. The height of this peak is smaller for the case of $N=12$, meaning that fewer particles in this system are making intercolumn jumps.

Another important factor that dictates how easy it is for particles to engage in intercolumn jumps is the particle bending flexibility. In the next section, we shall discuss the influence of this factor on the motion of particles in the columnar phase.

\section{EFFECT OF PARTICLE BENDING FLEXIBILITY}

To study the effect of bending flexibility on the motion of our rodlike particles, we carry out simulations with two values of contour length over persistence length ratio, $L / L_{p}=$ 0.31 and 0.09 . These values correspond to the length over persistence length ratio of the wild-type $f d$ virus and its mutant $f d Y 21 M$ [51]. The rattling and jumping kind of motion that is discussed in this paper has been observed in the columnar phase of the $f d$ virus, but not in that of the much stiffer mutant $f d Y 21 M$. This indicates that bending flexibility enhances the looplike motion of particles in the columnar phase. To investigate this further, we calculate the self-part of the Van Hove function for the simulations with the two values of $L / L_{p}$.

Shown in Fig. 6 is the component of the self-part of the Van Hove function parallel to the column axes, $G_{s}^{\|}$, for three system sizes with $N=12,50$, and 200 particles in each column and two values of the length over persistence length ratio $L / L_{p}=0.31$ and 0.09 . For all three system sizes, the more flexible particles with $L / L_{p}=0.31$ are more mobile. For the case of the smallest system with $N=12$ particles per column, a peak appears at $z / L=1$ for both values of $L / L_{p}$ [see Fig. 6(a)]. The height of the peak is smaller for the more rigid particles, confirming that particle flexing enhances the looplike motion of particles in the columnar phase. This is presumably because the free energy barrier between columns is reduced for more flexible particles, as these can bend and more effectively explore their direct environment, i.e., engaging in column-to-column jumps that trigger the looplike motion occurs more frequently for more flexible particles. 

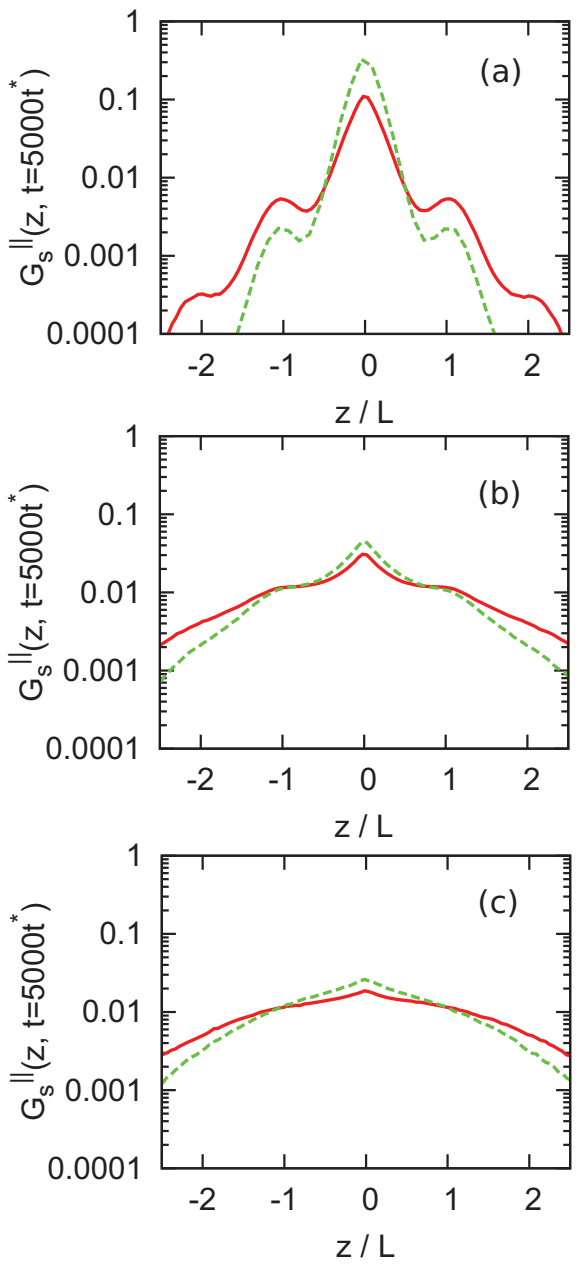

FIG. 6. (Color online) Component of the self-part of the Van Hove function parallel to the column axes, $G_{s}^{\|}(z, t)$, after a time interval of $t=5000 t^{*}$ obtained from simulations with (a) $N=12$, (b) $N=50$, and (c) $N=200$ particles per column for two values of length over persistence length ratio $L / L_{p}=0.31$ (red solid line) and $L / L_{p}=$ 0.09 (green dashed line). Here, $L$ is the length of a particle and $t^{*}$ is the simulation time unit. There are 100 columns in the simulation boxes with column-to-column distance of $d=1.75 \sigma$. The average linear fraction of particles in each column is $\psi=0.95$. The strength of the external potential is $k=1.5 k_{B} T$.

To check this, we obtain the component of the self-part of the Van Hove function perpendicular to the columns axes, $G_{s}^{\perp}(R, t)$. Shown in Fig. 7 is the component of $G_{s}$ perpendicular to the columns axes for the three system sizes and the two values of $L / L_{p}$. Again, for all three system sizes, the height of the peak on $R / d=1$ is larger for more flexible particles. This corroborates our suggestion that particle bending flexibility enhances the column-to-column jump probability. Interestingly, for the case of the smallest system, the difference between the height of the peaks corresponding to $L / L_{p}=0.09$ and 0.31 is larger than the other systems with $N=50$ and 200 particles per column. This is probably due to the large magnitude of fluctuations in the larger system sizes. As mentioned earlier, due to the presence of these fluctuations, voids spontaneously form in columns and this makes it easier for particles to jump from one column to another without
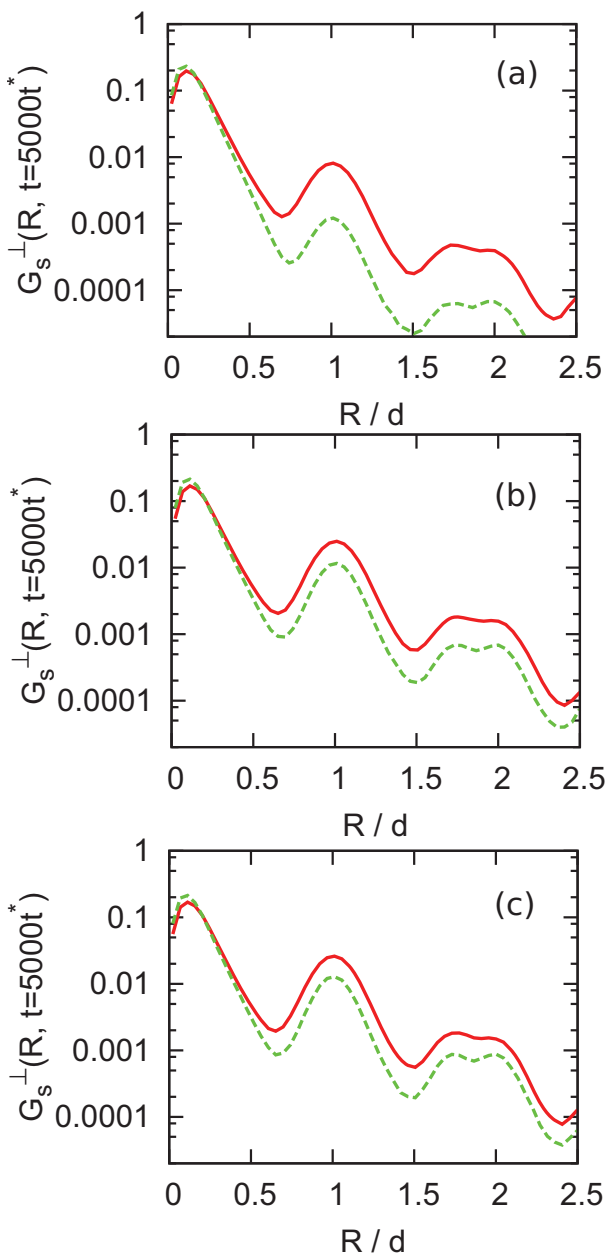

FIG. 7. (Color online) Component of the self-part of the Van Hove function parallel to the column axes, $G_{s}^{\perp}(R, t)$, after a time interval of $t=5000 t^{*}$ obtained from simulations with (a) $N=12$, (b) $N=50$, and (c) $N=200$ particles per column for two values of length over persistence length ratio $L / L_{p}=0.31$ (red solid line) and $L / L_{p}=0.09$ (green dashed line). Here, $L$ is the length of a particle and $t^{*}$ is the simulation time unit. There are 100 columns in the simulation boxes with column-to-column distance of $d=1.75 \sigma$. The average linear fraction of particles in each column is $\psi=0.95$. The strength of the external potential is $k=1.5 k_{B} T$.

actually having to bend too much. For the case of the smallest systems, however, the magnitude of the fluctuations is small and the formation of large enough voids in columns is a rare event. Therefore, particles need to bend in order to reach other columns. This means that by increasing the bending flexibility, the number of column-to-column jumps increases, especially for small systems.

\section{CONCLUSIONS AND OUTLOOK}

Inspired by recent experimental observations, we performed Brownian dynamic simulations in a dense columnar phase of hard filamentous particles [41]. We find that most particles rattle around their equilibrium positions, but that occasionally some of the particles engage in a rare loop-type collective motion. Some of the involved particles exchange columns, push other particles that stay in their columns 
forward, and force them to suddenly engage in a jumplike displacement. We obtained the distribution of jump lengths and observe that for sufficiently small system sizes, there is a peak on a full particle length. For larger systems, the width of the distribution increases and for sufficiently large systems, the peak disappears completely. This we identify to be due to the increase in the magnitude of the fluctuations in the positions of particles as the system becomes larger. We also investigated the effect of particle bending flexibility on the motion of particles in the columnar phase by performing simulations with two values of the persistence length of the particle and found that bending flexibility enhances the loop-type motion of particles in the columnar phase, especially for the smaller systems. In our simulations, we needed to stabilize the columnar phase in favor of the smectic phase, which for the small aspect ratio of 5.8 is the more stable phase, by making use of an external potential and an uneven boundary. Future work needs to be done to obtain the phase diagram of long filamentous particles and to investigate whether particle bending flexibility destabilizes the smectic phase and induces the formation of a columnar phase.

\section{ACKNOWLEDGMENTS}

The authors would like to thank S. Belli, Prof. R. van Roij, E. Grelet, and P. Lettinga for useful discussions. The work of S.N. forms part of the research program of the Dutch Polymer Institute (DPI, Project No. 698).
[1] G. J. Vroege and H. N. W. Lekkerkerker, Rep. Prog. Phys. 55, 1241 (1992).

[2] A. Stroobants, H. N. W. Lekkerkerker, and D. Frenkel, Phys. Rev. A 36, 2929 (1987).

[3] P. Davidson and J. C. P. Gabriel, Curr. Opin. Colloid Interface Sci. 9, 377 (2005).

[4] Z. Dogic and S. Fraden, Curr. Opin. Colloid Interface Sci. 11, 47 (2006).

[5] H. H. Wensink and H. N. W. Lekkerkerker, Mol. Phys. 107, 2111 (2009).

[6] F. M. van der Kooij, K. Kassapidou, and H. N. W. Lekkerkerker, Nature (London) 406, 868 (2000).

[7] A. Kuijk, A. van Blaaderen, and A. Imhof, J. Am. Chem. Soc. 133, 2346 (2011).

[8] L. Onsager, Ann. N.Y. Acad. Sci. 51, 627 (1949).

[9] Z. Dogic and S. Fraden, Langmuir 16, 7820 (2000).

[10] F. Tombolato, A. Ferrarini, and E. Grelet, Phys. Rev. Lett. 96, 258302 (2006).

[11] R. B. Meyer, in Dynamics and Patterns in Complex Fluids, edited by A. Onuki and K. Kawasaki (Springer, Berlin, 1990), Vol. 52.

[12] F. Livolant, Physica A 176, 117 (1991).

[13] X. Wen and R. B. Meyer, Phys. Rev. Lett. 59, 1325 (1987).

[14] D. Frenkel, H. N. W. Lekkerkerker, and A. Stroobants, Nature (London) 332, 822 (1988).

[15] Z. Dogic and S. Fraden, Phys. Rev. Lett. 78, 2417 (1997).

[16] X. Wen, R. B. Meyer, and D. L. D. Caspar, Phys. Rev. Lett. 63, 2760 (1989).

[17] M. S. Yu, V. P. Conticello, G. Zhang, C. Kayser, M. J. Fournier, T. L. Mason, and D. A. Tirrell, Nature (London) 389, 167 (1997).

[18] E. Grelet, Phys. Rev. Lett. 100, 168301 (2008).

[19] R. Podgornik, H. H. Strey, K. Gawrisch, D. C. Rau, A. Rupprecht, and V. A. Parsegian, Proc. Nat. Acad. Sci. 93, 4261 (1996).

[20] H. H. Strey, J. Wang, R. Podgornik, A. Rupprecht, L. Yu, V. A. Parsegian, and E. B. Sirota, Phys. Rev. Lett. 84, 3105 (2000).

[21] P. van der Schoot, J. Phys. II (France) 6, 1557 (1996).

[22] A. V. Tkachenko, Phys. Rev. Lett. 77, 4218 (1996).

[23] G. Cinacchi and L. De Gaetani, Phys. Rev. E 77, 051705 (2008).

[24] H. H. Wensink, J. Chem. Phys. 126, 194901 (2007).
[25] J. A. C. Veerman and D. Frenkel, Phys. Rev. A 43, 4334 (1991). [26] P. Bolhuis and D. Frenkel, J. Chem. Phys. 106, 666 (1997).

[27] R. P. Sear and G. Jackson, J. Chem. Phys. 102, 2622 (1995).

[28] S. Belli, A. Patti, R. van Roij, and M. Dijkstra, J. Chem. Phys. 133, 154514 (2010).

[29] B. J. Lemaire, P. Davidson, P. Panine, and J. P. Jolivet, Phys. Rev. Lett. 93, 267801 (2004).

[30] J. Käs, H. Strey, J. Tang, D. Finger, R. Ezzell, E. Sackmann, and P. Janmey, Biophys. J. 70, 609 (1996).

[31] H. Maeda and Y. Maeda, Phys. Rev. Lett. 90, 018303 (2003).

[32] M. P. Lettinga, E. Barry, and Z. Dogic, Europhys. Lett. 71, 692 (2005).

[33] E. Grelet, M. P. Lettinga, M. Bier, R. van Roij, and P. van der Schoot, J. Phys.: Condens. Matter 20, 494213 (2008).

[34] M. P. Lettinga and E. Grelet, Phys. Rev. Lett. 99, 197802 (2007).

[35] E. Pouget, E. Grelet, and M. P. Lettinga, Phys. Rev. E 84, 041704 (2011).

[36] M. Bier, R. van Roij, M. Dijkstra, and P. van der Schoot, Phys. Rev. Lett. 101, 215901 (2008).

[37] A. Kuijk, D. Byelov, A. Petukhov, A. van Blaaderen, and A. Imhof, Faraday Discuss. 159, 181 (2012).

[38] A. Patti, D. El Masri, R. van Roij, and M. Dijkstra, J. Chem. Phys. 132, 224907 (2010).

[39] R. Matena, M. Dijkstra, and A. Patti, Phys. Rev. E 81, 021704 (2010).

[40] A. Patti, D. El Masri, R. van Roij, and M. Dijkstra, Phys. Rev. Lett. 103, 248304 (2009).

[41] S. Naderi, E. Pouget, P. Ballesta, P. van der Schoot, M. P. Lettinga, and E. Grelet, Phys. Rev. Lett. 111, 037801 (2013).

[42] S. Naderi, and P. van der Schoot, J. Chem. Phys. (to be published).

[43] W. K. Kegel and A. van Blaaderen, Science 287, 290 (2000).

[44] A. R. Khokhlov and A. N. Semenov, Physica A 108, 546 (1981).

[45] A. R. Khokhlov and A. N. Semenov, Physica A 112, 605 (1982).

[46] H. Yamakawa and M. Fujii, J. Chem. Phys. 59, 6641 (1973).

[47] T. Odijk, Macromolecules 16, 1340 (1983).

[48] S. Plimpton, J. Comp. Phys. 117, 1 (1995).

[49] M. Doi and S. F. Edwards, The Theory of Polymer Dynamics (Oxford University Press, New York, 1988), Vol. 73.

[50] L. Van Hove, Phys. Rev. 95, 249 (1954).

[51] E. Barry, D. Beller, and Z. Dogic, Soft Matter 5, 2563 (2009). 